\title{
两种胺模板草酸铀酰配合物的合成、结构和光学 性质
}

乔雪玲 ${ }^{1}$, 王亚星 ${ }^{2}$, 何建刚 $^{1}$, 魏文琦 ${ }^{1}$, 葛瑞 ${ }^{1}$, 刘 春立 $^{1 *}$, 王伇凹 ${ }^{2 *}$

1. 北京分子科学国家实验室, 放射化学与辐射化学重点学科实验室, 北京大学化学与分子工程学院, 北京 100871

2. 苏州大学放射医学及交叉学科研究院, 苏州 215123

*通讯作者, E-mail: liucl@pku.edu.cn; shuaowang@suda.edu.cn

收稿日期: 2017-03-07; 接受日期: 2017-04-25; 网络版发表日期: 2017-08-29

国家自然科学基金(编号: 11475008, U1530112, 11075006, 91026010)资助项目

\begin{abstract}
摘要本文采用常温挥发法, 分别以质子化环已胺 $\left[\mathrm{C}_{6} \mathrm{H}_{14} \mathrm{~N}\right]^{+}$和 $N, N$-二甲基环已胺 $\left[\mathrm{C}_{8} \mathrm{H}_{18} \mathrm{~N}\right]^{+}$为模板剂, 草酸为配 体, 合成铀酰配合物 $\left[\mathrm{C}_{6} \mathrm{H}_{14} \mathrm{~N}\right]_{4}\left[\mathrm{UO}_{2}\left(\mathrm{C}_{2} \mathrm{O}_{4}\right)_{2}\left(\mathrm{HC}_{2} \mathrm{O}_{4}\right)\right] \mathrm{NO}_{3} \cdot 2 \mathrm{H}_{2} \mathrm{O}(\mathbf{C H A})$ 和 $\left[\mathrm{C}_{8} \mathrm{H}_{18} \mathrm{~N}\right]\left[\mathrm{UO}_{2}\left(\mathrm{C}_{2} \mathrm{O}_{4}\right)\left(\mathrm{NO}_{3}\right)\right](\mathbf{N N D M C H A})$. 通过单晶X射线衍射、粉末衍射(PXRD)、热重-差热(TG-DSC)、红外光谱(IR)、拉曼光谱(Raman)、紫外-可 见光谱(UV-Vis)和荧光光谱进行表征, 解析配合物结构并研究其光学性质. CHA 和 NNDMCHA均属于单斜晶 系, 空间群 $P 2_{1} / \mathrm{c}, \mathrm{UO}_{2}{ }^{2+}$ 分别为平面五配位和六配位. CHA 结构中结晶水通过 $\mathrm{O}-\mathrm{H} \cdots \mathrm{O}$ 氢键连接单核阴离子形 成沿 $c$ 方向延伸的 $\mathrm{s}$ 形阴离子链. 质子化胺通过 $\mathrm{N}-\mathrm{H} \cdots \mathrm{O}$ 和 $\mathrm{C}-\mathrm{H} \cdots \mathrm{O}$ 氢键连接阴离子链, 导向其分别形成较大 的六边形和较小的平行四边形孔道. 两种配合物都呈现出光致荧光减弱现象, 是潜在的荧光传感器材料.
\end{abstract}

关键词质子化胺, 模板, 草酸铀酰配合物, 光学性质

\section{1 引言}

草酸具有灵敏的氧化还原性、可调的酸碱性和 较弱的热稳定性等特性, 被广泛用于钣系领域的研究, 如钣系元素分离、净化或回收的沉淀剂、超铀原材 料生产中铀氧化物制备的前驱体等 ${ }^{[1]}$. 另外, 长久以来 合成的草酸铀酰杂化材料被作为一种优良的光辐射 探测计 ${ }^{[2]}$. 铀酰杂化材料因其丰富的拓扑结构、电学 性质、气体储存、药物传输和催化等特点和应用, 受 到广泛关注 ${ }^{[3]}$. 近十年, 草酸铀酰杂化材料的研究主 要是引入多配体, 如乙酸 ${ }^{[4]}$ 、过氧离子 ${ }^{[5]}$ 、硫酸和硫 氧根 ${ }^{[6]}$ 等; 引入异金属作为共同中心原子, 如 $\mathrm{Cu}^{[7]} 、 \mathrm{Eu}$

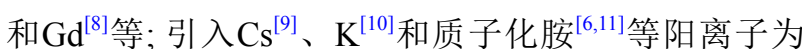
模板. 质子化胺与阴离子通过阳离子-阴离子相互作 用形成晶态固体, 是合成功能固体材料的常用方法 ${ }^{[12]}$, 其主要起模板导向、空隙填充和电荷平衡等作用, 并 可作为氢键给体或受体, 连接阴离子骨架, 使得化合 物形成高维数的复杂 $3 \mathrm{D}$ 晶体结构 ${ }^{[13]}$. 目前, 草酸铀酰 配合物中质子化胺对结构构筑影响的研究非常少.

本文设计合成两种质子化胺模板的草酸铀酰配 合物CHA和NNDMCHA, 并通过单晶衍射解析其晶体 结构, 通过红外光谱(IR)、拉曼光谱(Raman)、UV-Vis 和苂光等多种光谱手段研究其光谱, 通过热重研究其 热稳定性, 以期深入探索质子化胺对于晶体结构形成

引用格式: 乔雪玲, 王亚星, 何建刚, 魏文琦, 葛瑞, 刘春立, 王没. 两种胺模板草酸铀酰配合物的合成、结构和光学性质. 中国科学: 化学, 2017, 47: $1140-1147$

Qiao X, Wang Y, He J, Wei W, Ge R, Liu C, Wang S. Synthesis, structure and optical properties of two uranyl oxalate complexes templated by protonated amines. Sci Sin Chim, 2017, 47: 1140-1147, doi: 10.1360/N032017-00040 
的导向作用, 探究配合物的光学性质. 质子化胺模板 导向阴离子形成不同形状和大小的孔道结构, 并通过 氢键挂在孔道内壁上, 填充空隙. 两种草酸铀酰配合 物都呈现出光致荧光减弱现象, 推测可能是光照催化 草酸分解 ${ }^{[3,14]}$, 进而改变结构中铀酰离子的环境, 影响 荧光发射, 有望应用于荧光传感器材料 ${ }^{[3 b]}$.

\section{2 实验部分}

\section{1 试剂与仪器}

所用试剂均为分析纯, 未经进一步提纯. $\mathrm{UO}_{2}\left(\mathrm{NO}_{3}\right)_{2} \cdot 6 \mathrm{H}_{2} \mathrm{O}$ (中国医药公司北京化学试剂采购 供应站), 无水草酸(ACROS Organics 公司, 美国), 环 己胺和 $N, N$-二甲基环已胺 (北京百灵威科技有限公 司, 中国), 无水乙醇(中国西陇化工股份有限公司), 超 纯水.

Xcalibur Atlas Gemini ultra衍射仪(安捷伦科技公 司, 美国), RINT2000粉末X射线衍射仪 (Rigaku Denki Co., Ltd 公司, 日本), vario EL元素分析仪(Elementar Analysensysteme GmbH公司, 德国), Q600-SDT热重差 热同步测定仪(TA公司, 美国), NICOLET iN10 MX显微 红外光谱仪(Thermo Scientific 公司, 美国), HR800激光 拉曼测试平台(Jobin-Yvon Labram公司, 法国), CRAIC 科技显微分光光度计(CRAIC公司, 美国).

\section{2 合成方法}

$\left[\mathrm{C}_{6} \mathrm{H}_{14} \mathrm{~N}\right]_{4}\left[\mathrm{UO}_{2}\left(\mathrm{C}_{2} \mathrm{O}_{4}\right)_{2}\left(\mathrm{HC}_{2} \mathrm{O}_{4}\right)\right] \mathrm{NO}_{3} \cdot 2 \mathrm{H}_{2} \mathrm{O} \quad$ (CHA): $600 \mu \mathrm{L} 0.5 \mathrm{moL} / \mathrm{L}$ 环已胺水溶液与 $500 \mu \mathrm{L} 0.5 \mathrm{moL} / \mathrm{L}$ 草 酸水溶液于 $10 \mathrm{~mL}$ 玻璃瓶中混合均匀, 沿瓶壁缓慢加 入 $500 \mu \mathrm{L} 0.1 \mathrm{moL} / \mathrm{L}$ 硝酸铀酰水溶液, 封闭静置. $27 \mathrm{~d}$ 后收取黄绿色棒状晶体, 自然晾干, 产率为 $26.9 \%$. C、 $\mathrm{H} 、 \mathrm{~N}$ 元素分析结果(\%)为C 35.61, H 5.55, N 6.28; 计 算结果(\%)为 C $34.85, \mathrm{H} 5.90, \mathrm{~N} 6.78$.

$\left[\mathrm{C}_{8} \mathrm{H}_{18} \mathrm{~N}\right]\left[\mathrm{UO}_{2}\left(\mathrm{C}_{2} \mathrm{O}_{4}\right)\left(\mathrm{NO}_{3}\right)\right]$ (NNDMCHA): $500 \mu \mathrm{L}$ $0.5 \mathrm{moL} / \mathrm{L}$ 草酸水溶液与 $500 \mu \mathrm{L} 0.1 \mathrm{moL} / \mathrm{L}$ 硝酸铀酰水 溶液于 $10 \mathrm{~mL}$ 玻璃瓶中混合均匀, 沿瓶壁缓慢加入 $200 \mu \mathrm{L} 0.5 \mathrm{moL} / \mathrm{L} N, N$-二甲基环已胺乙醇溶液, 封闭 静置, $159 \mathrm{~d}$ 后, 收取黄绿色针状晶体 $\mathrm{UO}_{2} \mathrm{C}_{2} \mathrm{O}_{4} \cdot 3 \mathrm{H}_{2} \mathrm{O}$, 将 母液封口继续静置, $13 \mathrm{~d}$ 后, 收取黄绿色块状晶体, 无 水乙醇洗涤后, 自然晾干, 产率为 $35.8 \%$. C、 $\mathrm{H} 、 \mathrm{~N}$ 元 素分析结果(\%)为C $21.98, \mathrm{H} 3.35, \mathrm{~N}$ 5.07; 计算结果(\%)
为 C 21.90, H 3.28, N 5.11.

\section{3 表征方法}

晶体结构测试和解析: 配合物晶体的单晶 $\mathrm{X}$ 射线 衍射数据在恒温 $293 \mathrm{~K}$ 下收集, 入射波长为 $0.71073(\mathrm{Mo}$ $\mathrm{K} \alpha$ 辐射). 在 $\mathrm{Olex} 2^{[15]}$ 中, 分别通过 ShelXS 和ShelXL ${ }^{[16]}$ 采用直接法进行结构解析, 并用最小二乘法进行结 构精修. 除水分子外, 氢原子通过理论加氢得到. 氢 键和理论粉末 X射线衍射图谱通过Platon计算获得. 表 1 为化合物 CHA 和 NNDMCHA 的晶体学数据, 部 分键长和键角见表 2 . 粉末 $X$ 射线衍射数据: 制样和 压片后, 以 $4 \% \mathrm{~min}$ 的速率收集 $5^{\circ} \sim 60^{\circ}$ 范围内衍射数 据. CHA 和 NNDMCHA的 CCDC 号分别为 1545407 和 1545406.

热分析: 将化合物晶体样品在玛瑙研钵内研细, 取3 5 mg样品于氧化铝坩埚中, 在Q600-SDT热分析仪 上, 采用氮气气氛, 以 $5^{\circ} \mathrm{C} / \mathrm{min}$ 的速率升温至 $800^{\circ} \mathrm{C}$ 进 行热分解测试. 采用TA Universal Analysis 2000软件进 行数据分析和处理.

光谱分析: 均采用单晶进行测试. 显微红外光谱数 据范围为4000 $600 \mathrm{~cm}^{-1}$. 拉曼光谱激发波长为 $633 \mathrm{~nm}$. 紫外-可见吸收光谱和苂光发射光谱均通过显微分光 光度计收集数据, 后者激发波长为 $365 \mathrm{~nm}$.

表 1 化合物CHA和NNDMCHA的晶体学数据

\begin{tabular}{|c|c|c|}
\hline 化合物 & CHA & NNDMCHA \\
\hline 分子式 & $\mathrm{C}_{30} \mathrm{H}_{61} \mathrm{~N}_{5} \mathrm{O}_{19} \mathrm{U}$ & $\mathrm{C}_{10} \mathrm{H}_{18} \mathrm{~N}_{2} \mathrm{O}_{9} \mathrm{U}$ \\
\hline$f_{\mathrm{W}}$ & 1033.86 & 548.29 \\
\hline$T(\mathrm{~K})$ & 293(2) & $293(2)$ \\
\hline 晶系 & 单斜 & 单斜 \\
\hline 空间群 & $P 2_{1} / \mathrm{c}$ & $P 2_{1} / \mathrm{c}$ \\
\hline$a(\AA)$ & $11.9358(2)$ & $11.3688(6)$ \\
\hline$b(\AA)$ & $18.7279(3)$ & $11.0238(5)$ \\
\hline$c(\AA)$ & $19.1956(4)$ & $13.4478(8)$ \\
\hline$\alpha\left(^{\circ}\right)$ & 90 & 90 \\
\hline$\beta\left(^{\circ}\right)$ & $102.2096(19)$ & $112.465(6)$ \\
\hline$\gamma\left({ }^{\circ}\right)$ & 90 & 90 \\
\hline$V\left(\AA^{3}\right)$ & $4193.80(13)$ & $1557.48(15)$ \\
\hline$Z, D_{\mathrm{c}}\left(\mathrm{g} / \mathrm{cm}^{3}\right)$ & $4,1.637$ & $4,2.338$ \\
\hline$R_{1}^{\text {a) }}, w R_{2}{ }^{\text {b) }}[I \geq 2 \sigma(I)]$ & $0.0324,0.0613$ & $0.0357,0.0876$ \\
\hline
\end{tabular}

a) $\mathrm{R} 1=\Sigma|| F_{\mathrm{o}}|-| F_{\mathrm{c}}|/ \Sigma| F_{\mathrm{o}} \mid ;$ b) $w R_{2}=\left[\Sigma \mathrm{w}\left(F_{\mathrm{o}}{ }^{2}-F_{\mathrm{c}}{ }^{2}\right)^{2}\right] / \Sigma w\left(F_{\mathrm{o}}{ }^{2}\right]^{1 / 2}$ 
表 2 CHA 和NNDMCHA的部分键长 $(\AA)$ 和键角 $\left({ }^{\circ}\right)$

\begin{tabular}{|c|c|c|}
\hline 化合物 & CHA & NNDMCHA \\
\hline$T(\mathrm{~K})$ & $293(2)$ & 293(2) \\
\hline $\mathrm{U}-\mathrm{O}_{\mathrm{ax}}$ & $1.759(2), 1.763(2)$ & $1.747(4), 1.754(4)$ \\
\hline $\mathrm{U}-\mathrm{O}_{\mathrm{eq}}$ & $2.346(2) \sim 2.400(2)$ & $2.473(5) \sim 2.527(5)$ \\
\hline $\mathrm{C}-\mathrm{O}$ & $1.198(4) \sim 1.293(4)$ & $1.243(8) \sim 1.262(8)$ \\
\hline $\mathrm{N}-\mathrm{O}$ & $1.221(4) \sim 1.266(4)$ & $1.210(8) \sim 1.270(9)$ \\
\hline $\mathrm{C}-\mathrm{C}$ & $1.427(8) \sim 1.550(5)$ & $1.484(13) \sim 1.519(12)$ \\
\hline $\mathrm{N}-\mathrm{C}$ & $1.489(4) \sim 1.495(5)$ & $1.485(12) \sim 1.541(9)$ \\
\hline $\mathrm{O}_{\mathrm{ax}}-\mathrm{U}-\mathrm{O}_{\mathrm{ax}}$ & $178.28(11)$ & $178.9(2)$ \\
\hline $\mathrm{O}_{\mathrm{ax}}-\mathrm{U}-\mathrm{O}_{\mathrm{eq}}$ & $86.99(11) \sim 92.14(10)$ & $83.0(2) \sim 97.9(2)$ \\
\hline $\mathrm{O}_{\mathrm{eq}}-\mathrm{U}-\mathrm{O}_{\mathrm{eq}}$ & $65.85(7) \sim 157.48(8)$ & 49.71(19) 172.23(16) \\
\hline $\mathrm{U}-\mathrm{O}-\mathrm{C}(\mathrm{ox})$ & $121.89(19) \sim 132.8(2)$ & $120.7(4) \sim 121.3(4)$ \\
\hline $\mathrm{U}-\mathrm{O}-\mathrm{N}$ & - & 97.9(4), 97.4(4) \\
\hline $\mathrm{O}-\mathrm{C}-\mathrm{O}(\mathrm{ox})$ & $125.8(3) \sim 127.1(3)$ & $126.6(6), 126.7(6)$ \\
\hline $\mathrm{O}-\mathrm{C}-\mathrm{C}(\mathrm{ox})$ & $114.0(3) \sim 120.2(3)$ & $116.3(8) \sim 117.0(8)$ \\
\hline $\mathrm{O}-\mathrm{N}-\mathrm{O}$ & $119.6(3) \sim 120.5(4)$ & $114.3(6) \sim 123.0(8)$ \\
\hline $\mathrm{C}-\mathrm{N}-\mathrm{C}$ & - & $110.9(7) \sim 115.5(6)$ \\
\hline $\mathrm{N}-\mathrm{C}-\mathrm{C}$ & 109.3(3) 114.0(4) & $110.9(7), 112.4(6)$ \\
\hline $\mathrm{C}-\mathrm{C}-\mathrm{C}$ & $109.5(3) \sim 114.7(4)$ & $107.7(6) \sim 113.3(8)$ \\
\hline $\mathrm{C}-\mathrm{H} \cdots \mathrm{O}$ & $3.488(4), 164.0$ & $\begin{array}{c}3.418(11), 3.450(11) \\
156.9,163.9\end{array}$ \\
\hline $\mathrm{C}-\mathrm{H} \cdots \mathrm{N}$ & $3.590(6), 155.6$ & - \\
\hline $\mathrm{N}-\mathrm{H}^{\cdots} \cdots$ & $\begin{array}{c}2.791(5) \sim 3.426(4) \\
113.5 \sim 173.6\end{array}$ & $\begin{array}{c}2.841(8), 3.163(8) \\
167.1,121.4\end{array}$ \\
\hline $\mathrm{O}-\mathrm{H} \cdots \mathrm{N}$ & $3.267(4), 143(4)$ & - \\
\hline $\mathrm{O}-\mathrm{H} \cdots \mathrm{O}$ & $\begin{array}{c}2.714(4) \sim 3.174(5) \\
116(4) \sim 178(5)\end{array}$ & - \\
\hline
\end{tabular}

\section{3 结果与讨论}

\section{1 化合物晶体结构}

CHA和NNDMCHA都属于单斜晶系, 空间群 $P 2_{1} / \mathrm{c}$ (表 1). CHA 的单核阴离子 $\left[\mathrm{UO}_{2}\left(\mathrm{C}_{2} \mathrm{O}_{4}\right)_{3}\left(\mathrm{HC}_{2} \mathrm{O}_{4}\right)\right]^{3-}$ 由 $\mathrm{UO}_{2}{ }^{2+}$ 与两个 $\mu_{2}-\mathrm{Ox}$ (两个顺式氧原子参与鳌合配位, 简称 $c i s-0$ ) 及一个 $\mu_{1}$-Hox配位形成UO7五角双锥结构, $\mu_{1}-\mathrm{Hox}$ 朝轴向弯曲 $\left(\mathrm{U}-\mathrm{O} 18-\mathrm{C} 16\right.$ 键角为 $\left.132.81^{\circ}\right)$. 局部 环境如图1(a)所示, 阴离子通过 $\mathrm{N}-\mathrm{H} \cdots \mathrm{O}$ 和 $\mathrm{C}-\mathrm{H} \cdots \mathrm{O}$ 氢 键连接结晶水和模板质子化胺, $\mathrm{N} \cdots \mathrm{O}$ 距离为 2.791(5) $\sim 3.092(4) \AA, \mathrm{N}-\mathrm{H} \cdots \mathrm{O}$ 键角为 $113.5^{\circ} \sim 173.6^{\circ} ; \mathrm{C} \cdots \mathrm{O}$ 距离为 $3.488(4) \AA, \mathrm{C}-\mathrm{H} \cdots \mathrm{O}$ 键角为 $164.0^{\circ}$. 其中一个 $\mathrm{N} 31 \mathrm{H}_{3}{ }^{+}$以 $\mathrm{N}-\mathrm{H} \cdots \mathrm{O}$ 氢键连接 $\mu_{2}-\mathrm{ox}(\mathrm{O} 5)$ 和水分子 $(\mathrm{O} 10)$, 并与 O10- $\mathrm{H} \cdots$ O6 氢键共同形成八元环氢键模式, $\mathrm{R}_{3}{ }^{3}(8)^{[17]}$. 两个 $\mathrm{N} 45 \mathrm{H}_{3}{ }^{+}$和一个 $\mathrm{N} 52 \mathrm{H}_{3}{ }^{+}$分别与 $\mathrm{ox}(c i s-\mathrm{o})$ 通
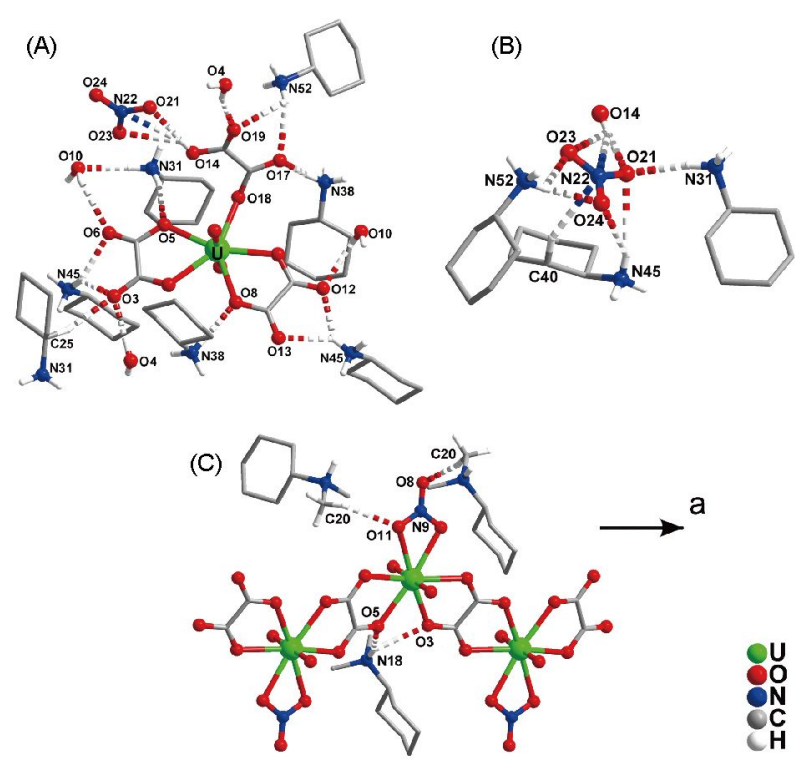

图 1 (A)CHA的阴离子局域环境; (B) $\mathrm{CHA}$ 中 $\mathrm{NO}_{3}{ }^{-}$的局域 环境; (C) NNDMCHA的阴离子链局域环境(网络版彩图)

过 $\mathrm{N}-\mathrm{H} \cdots \mathrm{O}$ 氢键相连, 形成双受体五元环氢键模式, $\mathrm{R}_{1}^{2}(5)^{[17]}$. 不同的是, 两个 $\mathrm{N}_{4} 5 \mathrm{H}_{3}{ }^{+}$连接的是 $\mu_{2}-\mathrm{ox}$, 而 $\mathrm{N} 52 \mathrm{H}_{3}{ }^{+}$连接的是 $\mu_{1}-\mathrm{Hox} . \mu_{1}-\mathrm{Hox}$ 的O14H以 $\mathrm{O}-\mathrm{H} \cdots \mathrm{O}$ 和 $\mathrm{O}-\mathrm{H} \cdots \mathrm{N}$ 氢键通过三受体氢键模式连接游离 $\mathrm{NO}_{3}{ }^{-}(\mathrm{N} 22$, $\mathrm{O} 21, \mathrm{O} 23), \mathrm{O} \cdots \mathrm{O}$ 距离为 $2.693(4) / 3.007(4) \AA, \mathrm{O}-\mathrm{H} \cdots \mathrm{O}$ 键角为 $167(5)^{\circ} / 116(4)^{\circ} ; \mathrm{O} \cdots \mathrm{N}$ 距离为 $3.267(4) \AA, \mathrm{O}-\mathrm{H} \cdots$ $\mathrm{N}$ 键角为 $143(4)^{\circ}$. 此外, 游离 $\mathrm{NO}_{3}{ }^{-}$还作为氢键受体与 3 个质子化环已胺相连, $\mathrm{N}_{2} 2 \mathrm{H}_{3}{ }^{+}$和 $\mathrm{N}_{4} 5 \mathrm{H}_{3}{ }^{+}$均以 $\mathrm{N}-\mathrm{H} \cdots$ $\mathrm{O}$ 氢键分别与 $\mathrm{NO}_{3}{ }^{-}(\mathrm{O} 23, \mathrm{O} 24)$ 和 $\mathrm{NO}_{3}{ }^{-}(\mathrm{O} 21, \mathrm{O} 24)$ 形成 四元环双受体氢键模式, $R_{1}{ }^{2}(4)$ (图1(B) $)^{[17]}$. 含 $N 45$ 的 $\left[\mathrm{C}_{6} \mathrm{H}_{14} \mathrm{~N}\right]^{+}$同时通过 $\mathrm{C}_{40}-\mathrm{H} \cdots \mathrm{N}_{22}$ 氢键与 $\mathrm{NO}_{3}{ }^{-}$连接, $\mathrm{C} \cdots$ $\mathrm{N}$ 距离为 $3.590(6) \AA, \mathrm{C}-\mathrm{H} \cdots \mathrm{N}$ 键角为 $155.6^{\circ}$. 阳离子 $\left[\mathrm{C}_{6} \mathrm{H}_{14} \mathrm{~N}\right]^{+}$在结构中共有4种配位环境(图1(A, B)). 对于 NNDMCHA, $\mathrm{UO}_{2}{ }^{2+}$ 采取六配位方式, 与两个 $\mu_{2}-\mathrm{ox}(c i s-\mathrm{o})$ 及一个 $\mu_{2}-\mathrm{NO}_{3}{ }^{-}$形成六角双锥多面体, 并通过 $\mu_{4}-\mathrm{ox}$ 桥 连形成沿 $a$ 方向的阴离子长链(图 1(C)), $\mathrm{NO}_{3}^{-}$沿长链 呈上下间隔分布, 配位 $\mathrm{ox}$ 和 $\mathrm{NO}_{3}{ }^{-}$分别通过 $\mathrm{N}-\mathrm{H} \cdots \mathrm{O}$ 和 $\mathrm{C}-\mathrm{H} \cdots \mathrm{O}$ 氢键与质子化胺 $\left[\mathrm{C}_{8} \mathrm{H}_{18} \mathrm{~N}\right]^{+}$相连, 前者形成环 状氢键模式 $R_{1}^{2}(4)^{[17]}$, 质子化胺仅有一种配位方式.

CHA 的单核阴离子在晶体结构中呈三维排列 (图2(A, B)). 阴离子单元和结晶水在 $b c$ 面均呈 4 种朝 向(图2(A)). 结晶水以 $\mathrm{O}-\mathrm{H} \cdots \mathrm{O}$ 氢键桥连相邻阴离子单 元的 $\mu_{2}-\mathrm{ox}(c i s-0)$ 和 $\mu_{1}-\mathrm{Hox}$, 形成 $c$ 方向的 $\mathrm{s}$ 形链, 链的弯 

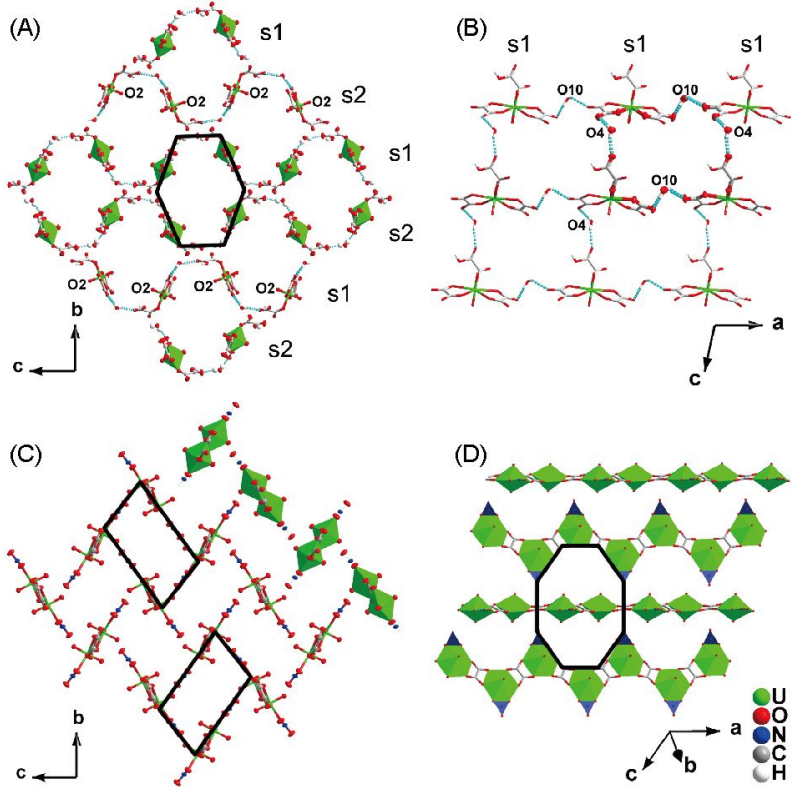

图 2 (A) $b c$ 面上, CHA的阴离子排列分布; (B) $a c$ 面上, CHA 的同向 $\mathrm{s}$ 形阴离子链网络结构; (C) $b c$ 面上, NNDMCHA的阴 离子链排列分布; (D) 相邻异向阴离子链的位置关系图(网 络版彩图)

曲来源于结晶水的 $\mathrm{O}-\mathrm{H}-\mathrm{O}$ 键角 $107.63^{\circ}$ 和阴离子中 $\mathrm{U}-\mathrm{O}_{\mu_{1}-\mathrm{Hox}}-\mathrm{C}_{\mu_{1}-\mathrm{Hox}}$ 键角 $132.81^{\circ}$ (朝轴向 $\mathrm{O} 2$ 弯曲), 且一条 链中结晶水和阴离子均只呈两种朝向, 不同朝向间隔 排列. $b$ 方向上, 相邻 $\mathrm{s}$ 形链 $\mathrm{s} 1$ 和 $\mathrm{s} 2$ 的单核阴离子和结晶 水朝向均不同, 4 种朝向的两种围成一个六边形环状孔 道. 同种 $\mathrm{s}$ 形链沿 $a$ 方向与同向结晶水通过 $\mathrm{O}-\mathrm{H} \cdots \mathrm{O}$ 氢 键桥连, 形成基于 $\mathrm{R}_{4}{ }^{8}(40)$ 环形氢键 ${ }^{[17]}$ 的二维阴离子骨 架网络结构(图2(B)). NNDMCHA的一维阴离子链在 $b c$ 面呈两种朝向交错排列, 围成平行四边形孔道(图2(C)), 相邻同向阴离子链则是围成八边形孔道, 包裹着中间 异向阴离子链的两个六角双锥多面体(图2(D)).

CHA 中的 $\mathrm{N}_{3} 8 \mathrm{H}_{3}{ }^{+} 、 \mathrm{~N}^{2} 1 \mathrm{H}_{3}{ }^{+} 、 \mathrm{C} 25 \mathrm{H}_{2}$ 和结晶水以 $\mathrm{N}-\mathrm{H} \cdots \mathrm{O} 、 \mathrm{O}-\mathrm{H} \cdots \mathrm{O}$ 和 $\mathrm{C}-\mathrm{H} \cdots \mathrm{O}$ 氢键桥连 $b$ 方向相邻 异向阴离子单元(图3(A)), 含 $\mathrm{R}_{6}{ }^{5}(18) 、 \mathrm{R}_{3}{ }^{3}(10) 、 \mathrm{R}_{3}{ }^{3}(8)$ 环形氢键模式 ${ }^{[17]}$, 使得沿 $c$ 方向 $s$ 形延展的二维阴离子 骨架沿 $b$ 方向连接堆积成3D结构 (图3(C)), 形成沿 $a$ 方 向伸展的六边形孔道. NNDMCHA的同向和异向相邻 阴离子链间由 $\mathrm{N} 18 \mathrm{H}_{3}{ }^{+}$和 $\mathrm{C}^{2} 2 \mathrm{H}_{3}$ 以 $\mathrm{N}-\mathrm{H} \cdots \mathrm{O}$ (双受体, $\mathrm{O} 5$ 和O3)和 $\mathrm{C}-\mathrm{H} \cdots \mathrm{O}$ 氢键(双受体, $\mathrm{O} 8$ 和O11)连接(图3(B)), 形成3D堆积结构 (图 3(D)), 含环形氢键模式 $\mathrm{R}_{4}{ }^{4}(22)$ 和 $\mathrm{R}_{4}{ }^{4}(13)^{[17]}$, 其中 $\mathrm{N}-\mathrm{H} \cdots \mathrm{O}$ 氢键的双受体氧来自一个六
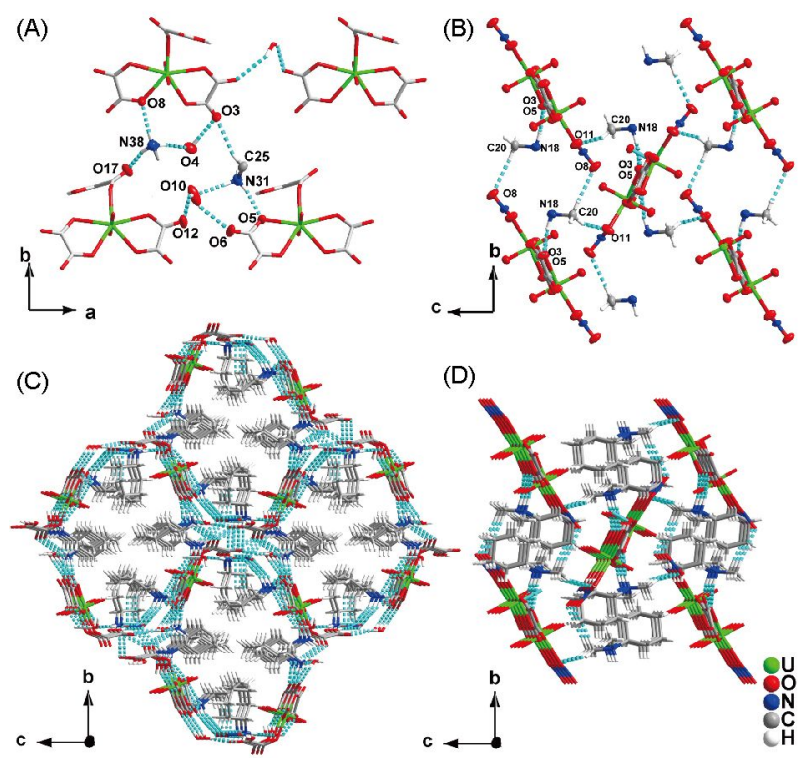

图 3 (A) $a b$ 面上, $\mathbf{C H A}$ 的异向阴离子链间氢键连接图; (B) NNDMCHA的阴离子链间氢键连接网络结构; (C, D) CHA 和NNDMCHA沿 $a$ 方向的3D堆积图(网络版彩图)

角双雉阴离子单元, 而 $\mathrm{C}-\mathrm{H} \cdots \mathrm{O}$ 氢键的双受体氧则来 自相邻异向阴离子链上的 $\mathrm{NO}_{3}^{-}$. CHA 和 NNDMCHA 化合物结构中的六边形和平行四边形孔道分别由阳 离子质子化胺 $\left[\mathrm{C}_{6} \mathrm{H}_{14} \mathrm{~N}\right]^{+}$(8组排列) 和 $\left[\mathrm{C}_{8} \mathrm{H}_{18} \mathrm{~N}\right]^{+}$(2组排 列)填充.

两种化合物阴离子孔道形状和大小及其3D堆积 结构和致密程度不同, 是由于模板阳离子质子化胺不 同, 导致其阴离子局部环境差异和阳离子-阴离子堆积 方式的不同. $\left[\mathrm{C}_{6} \mathrm{H}_{14} \mathrm{~N}\right]^{+}$以 $\mathrm{NH}_{3}{ }^{+}$为主参与形成氢键, 使 模板挂在阴离子孔道内壁上, 仅 $1 / 4$ 阳离子中紧邻 $\mathrm{NH}_{3}{ }^{+}$ 的一个 $\mathrm{CH}_{2}$ 同时参与氢键构筑. $\left[\mathrm{C}_{6} \mathrm{H}_{14} \mathrm{~N}\right]^{+}$中的芳香环 刚性较大, 且8组模板同处于孔道内部, 因而孔道较 大. $\left[\mathrm{C}_{8} \mathrm{H}_{18} \mathrm{~N}\right]^{+}$则是 $\mathrm{O} \cdots \mathrm{H}-\mathrm{N}-\mathrm{C}-\mathrm{H} \cdots \mathrm{O}$ 和 $\mathrm{O} \cdots \mathrm{H}-\mathrm{C}-\mathrm{H} \cdots$ $\mathrm{O}$ 直接连接同向平行和异向交错的相邻阴离子链, 因 而形成较小的平行四边形孔道, 进而构筑了更为致密 的晶体结构. 图4为化合物 CHA 和 NNDMCHA的粉末 衍射图谱, 基于结构数据计算的图谱与实验图谱吻合 度较高, 表明产品较纯。

\section{2 热分解性质}

CHA 在氮气气氛下的热分解曲线(TGA 和 DSC) 如图5所示. 由 TGA曲线推测该系列化合物的热失重 大致分为两个主要过程: 失水过程与无水中间相的热 


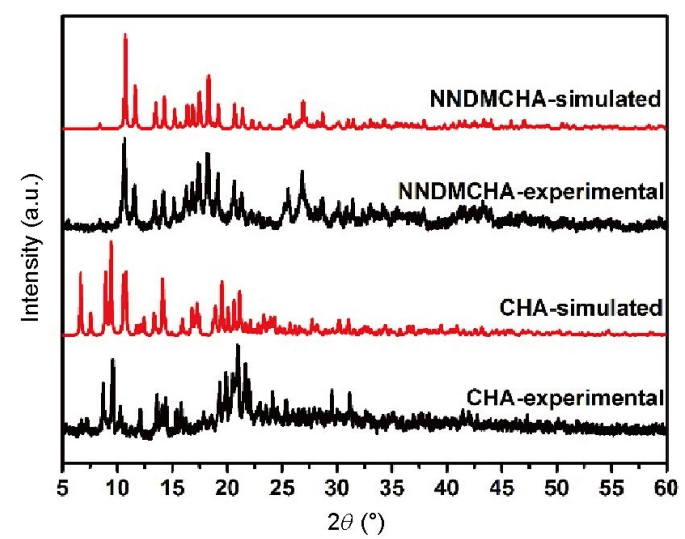

图 4 CHA 和NNDMCHA的粉末衍射图. experimental为实 验所得; simulated为基于单晶结构数据的理论拟合所得(网 络版彩图)

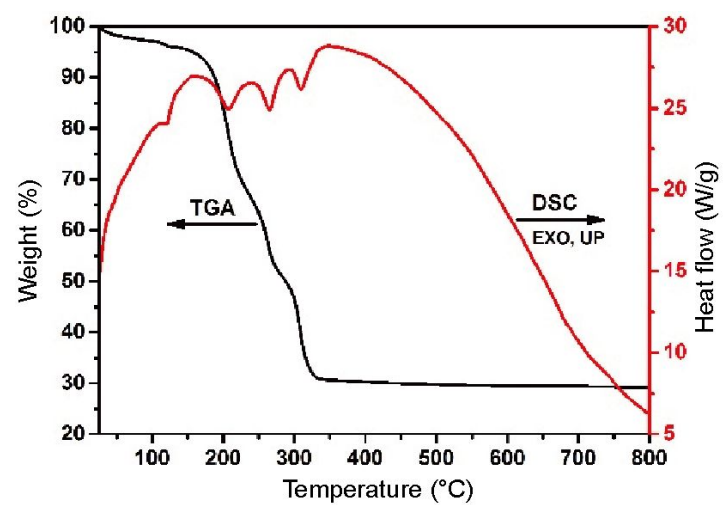

图 5 CHA的TGA-DSC曲线(网络版彩图)

分解过程. 第一个过程为连续失水, 约 $121^{\circ} \mathrm{C}$ 在 DSC曲 线上出现明显吸热峰, 吸热约 $50 \mathrm{~kJ} / \mathrm{mol}$, 总失重为 $3.4 \%$ (calc. 3.4\%). 第二个过程涉及三步连续热分解, 对应 207、 265 和 $309^{\circ} \mathrm{C}$ 附近的吸热, 共吸热约 $966 \mathrm{~kJ} / \mathrm{mol}$, 约 $350^{\circ} \mathrm{C}$ 基本完成, 该过程对应草酸和有机胺的解离, 产 物为 $\mathrm{UO}_{2} \mathrm{CO}_{3}{ }^{[18]}$, 总失重 $65.4 \%$ (calc. $65.4 \%$ ). 温度继续 升高时进一步失重, $800^{\circ} \mathrm{C}$ 时余烬 $29.2 \%$ (calc. $26.9 \%$, 按 $\mathrm{U}_{2} \mathrm{O}_{5}$ 计算 ${ }^{[18 \mathrm{c}]}$, 失重偏差可能是由于升温速度较快, 部分 $\mathrm{UO}_{2} \mathrm{CO}_{3}$ 并未完全分解, 并经 $\mathrm{UO}_{3}$ 中间产物, 转化 为 $\mathrm{U}_{2} \mathrm{O}_{5}$. NNDMCHA 因晶体生长周期长, 且产量非常 低, 没有进行热分解性质测试.

\section{3 光学性质}

CHA 和NNDMCHA的红外光谱和拉曼光谱相互 补充, 构成化合物各组分的特征吸收(图6). IR谱均包

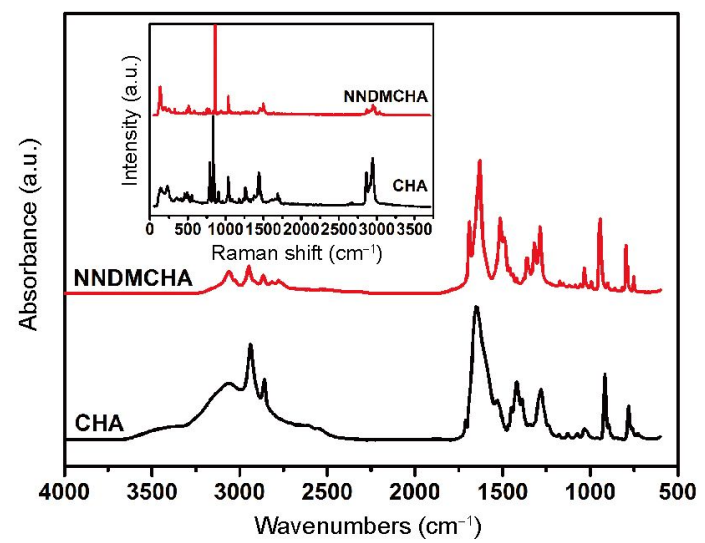

图 6 CHA和NNDMCHA的IR和Raman图谱(网络版彩图)

含 $\mathrm{C}-\mathrm{H} / \mathrm{N}-\mathrm{H}$ 伸缩振动 $\left(3500 \sim 2700 \mathrm{~cm}^{-1}\right), \mathrm{O}-\mathrm{C}=\mathrm{O} / \mathrm{C}-$ $\mathrm{C} / \mathrm{C}-\mathrm{N} / \mathrm{N}-\mathrm{O}$ 伸缩 振动和 $\mathrm{O}-\mathrm{C}=\mathrm{O}$ 弯曲振动 $\left(1800 \sim 1000 \mathrm{~cm}^{-1}\right)$ 以及 $\mathrm{UO}_{2}{ }^{2+}$ 的特征伸缩振动、 $\mathrm{O}-\mathrm{C}=\mathrm{O}$ 面内弯曲振动(950 750 $\left.\mathrm{cm}^{-1}\right)$. CHA 由于含结晶水, 因 而在 $3500 \mathrm{~cm}^{-1}$ 处出现 $\mathrm{H}_{2} \mathrm{O}$ 的宽吸收带. $\mathrm{O}=\mathrm{U}=\mathrm{O}$ 的不对 称伸缩振动 $\left(v_{\mathrm{as}}\right)$ 是 IR活性, 对称伸缩振动 $\left(v_{\mathrm{s}}\right)$ 是 Raman 活性, 但一些结构中铀酰的非对称环境会导致相应 弱 $v_{\mathrm{s}}$ 出现在IR 谱中 ${ }^{[19]}$. CHA 和 NNDMCHA 的IR 谱中 $\mathrm{O}=\mathrm{U}=\mathrm{O}$ 的 $v_{\mathrm{as}}$ 分别位于 916 和 $943 \mathrm{~cm}^{-1}$, 表明后者的轴 向 $\mathrm{U}-\mathrm{O}$ 键较强, 而赤道面的 $\mathrm{U}-\mathrm{O}$ 键相对较弱 ${ }^{[196,20]}$, 这 与后者 $\mathrm{U}-\mathrm{O}_{\mathrm{ax}}$ 键长较短, 而 $\mathrm{U}-\mathrm{O}_{\mathrm{eq}}$ 相对较长吻合 (表2), 也与 Raman谱中 NNDMCHA 的 $\mathrm{O}=\mathrm{U}=\mathrm{O}$ 对称伸缩振动 吸收峰 $862 \mathrm{~cm}^{-1}$ 处于比 $835 \mathrm{~cm}^{-1}$ (CHA) 更高频的位置 一致. 这两种化合物分别在红外光谱 $844 \mathrm{~cm}^{-1}$ (CHA) 和 $858 \mathrm{~cm}^{-1}$ (NNDMCHA) 位置出现 $\mathrm{O}=\mathrm{U}=\mathrm{O}_{\text {的弱 }} v_{\mathrm{s}}$ 峰.

CHA 和 NNDMCHA的固态UV-Vis吸收光谱非常 相似(图7), 350 500 nm有10个峰是典型的 $\mathrm{UO}_{2}{ }^{2}{ }^{+}$吸收峰, 对应激发态 $\mathrm{O}=\mathrm{U}=\mathrm{O}$ 的对称伸缩振动的叠加 ${ }^{[14,21]}$. 其中, 350 430 nm 的吸收峰归属为 ${ }^{1} \Sigma_{\mathrm{g}} \rightarrow^{+}{ }^{1} \Phi \mathrm{g}$ 与 ${ }^{1} \Sigma_{\mathrm{g}}{ }^{+} \rightarrow{ }^{1} \Delta \mathrm{g}$ 的跃 迁, 而430 500 nm的吸收峰归属为 ${ }^{1} \Sigma_{\mathrm{g}}{ }^{+} \rightarrow \Pi_{\mathrm{g}}$ 与 ${ }^{1} \Sigma_{\mathrm{g}}{ }^{+} \rightarrow{ }^{1} \Delta_{\mathrm{g}}$ 的跃迁 ${ }^{[21 b, 21 c]}$. 与CHA相比, NNDMCHA的UV-Vis 吸收 谱向高波数区蓝移, 这是由于赤道面 $\mathrm{U}-\mathrm{O}$ 越长, 轴向 $\mathrm{U}-\mathrm{O}$ 越短, $\mathrm{UO}_{2}{ }^{2+}$ 的 $v_{\mathrm{s}}$ 蓝移, 进而 UV-Vis 吸收光谱向高 波数区蓝移, 与Raman结果一致.

以 $365 \mathrm{~nm}$ 波长持续激发获得两种化合物的荧光 发射光谱变化图(图8(a,b)), 均呈现绿色苂光. 铀酰配 合物的苂光发射光谱是从 $\mathrm{UO}_{2}{ }^{2+}$ 的最低激发态 ${ }^{3} \Pi_{\mathrm{u}}$ 到基 态不同振动能级之间的跃迁 ${ }^{[21 a, 22]}$, 耦合了基态 $\mathrm{UO}_{2}{ }^{2+}$ 的 对称伸缩振动 ${ }^{[14,23]}$. CHA 和 NNDMCHA 的荧光发射 


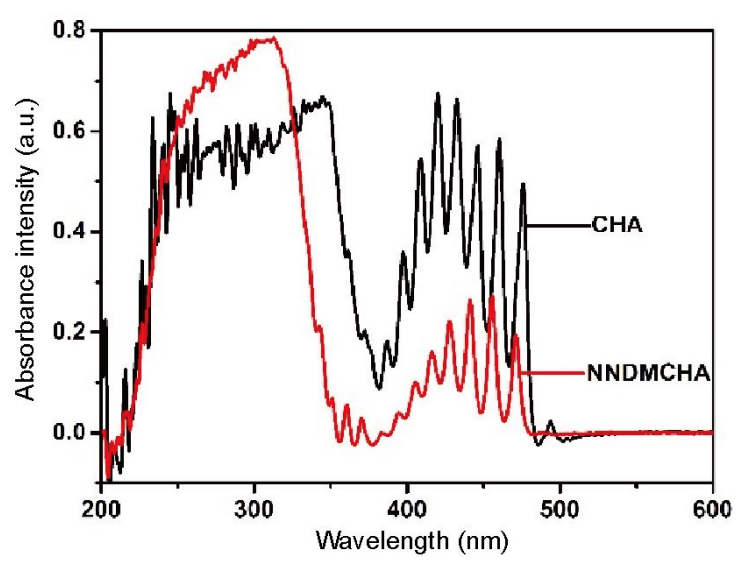

图 7 CHA 和NNDMCHA的UV-Vis光谱(网络版彩图)
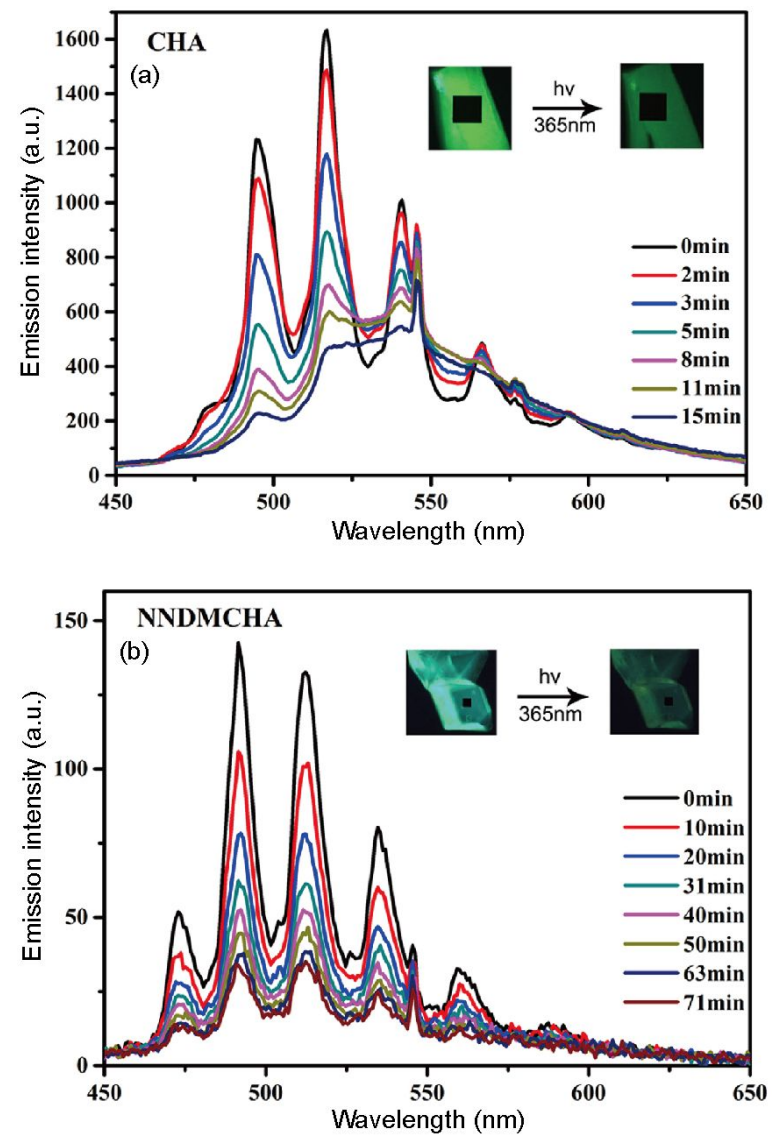

图 $8365 \mathrm{~nm}$ 波长下, CHA 和NNDMCHA的苂光发射光谱 变化图(网络版彩图)
峰分别位于：477、494、517、541 (545 shoulder)、 566 (577 shoulder)、594和 $611 \mathrm{~nm}$, 平均间距 $766 \mathrm{~cm}^{-1}$; $473 、 491 、 512 、 535$ (545 shoulder)、560和 $589 \mathrm{~nm}$, 平 均间距 $833 \mathrm{~cm}^{-1}$ (均为 $0 \mathrm{~min}$ 照射时光谱). 铀酰化合物 的苂光峰间距取决于配体偏离 $\mathrm{UO}_{2}{ }^{2+}$ 赤道面的程度, 偏离程度越小, 苂光峰的间距越大 ${ }^{[21 a, 22 e]}$, NNDMCHA 的荧光发射峰平均间距更大, 与单晶结构解析结果 一致. 此外, 两种铀酰配合物的苂光光谱与紫外可见 吸收光谱的 455 500 nm 存在镜像关系 ${ }^{[24]}$, 即荧光光谱 中476、494 nm (CHA)和471 nm (NNDMCHA)处的峰 对应于UV-Vis吸收光谱477、494 nm (CHA) 和 $473 \mathrm{~nm}$ (NNDMCHA) 的峰, 说明吸收光谱和苂光发射光谱起 源于相同的电子跃迁, 分别耦合了电子激发态的振动 能级和基态的振动能级.

从二者的苂光光谱变化图可知, 随照射时间延长, 绿色荧光均逐渐减弱. CHA 和NNDMCHA分别在照射 $15 \mathrm{~min}$ 和71 $\mathrm{min}$ 后, 470 520 $\mathrm{nm}$ 内的 3 个荧光发射峰强 度平均下降 $73 \%$. 推测可能是光照催化草酸分解 ${ }^{[3 b, 14]}$, 使得晶格层间距离、质子化胺阳离子位置或仅是铀 酰的配位环境等改变, 进而改变结构中铀酰离子的环 境, 影响荧光发射. 具有该光致荧光改变现象的配合 物有望应用于苂光传感器材料 ${ }^{[3 b]}$.

\section{4 结论}

采用常温挥发法, 合成了两种质子化胺模板的草 酸铀酰配合物CHA和NNDMCHA, 均属于单斜晶系, 空间群 $P 2_{1} / \mathrm{c}$, 阴离子单元分别为五角双雉 $(\mathbf{C H A})$ 和六 角双雉 (NNDMCHA) 结构. 模板 $\left[\mathrm{C}_{6} \mathrm{H}_{14} \mathrm{~N}\right]^{+}$和 $\left[\mathrm{C}_{8} \mathrm{H}_{18} \mathrm{~N}\right]^{+}$ 通过 $\mathrm{N}-\mathrm{H} \cdots \mathrm{O}$ 和 $\mathrm{C}-\mathrm{H} \cdots \mathrm{O}$ 氢键连接阴离子链, 使其分 别形成较大的六边形和较小的平行四边形孔道. 质子 化胺填充空隙, 挂在孔道内壁上. CHA的结构中结晶 水通过 $\mathrm{O}-\mathrm{H} \cdots \mathrm{O}$ 氢键也参与到阴离子骨架的构筑中. CHA 的热分解曲线主要包含失水与无水中间相的热 分解两个过程, 两个过程结束后分解为 $\mathrm{UO}_{2} \mathrm{CO}_{3}$. 两种 草酸铀酰配合物都表现出光致苂光减弱现象, 推测光 照可能催化结构中的草酸分解, 致使铀酰离子的环境 发生改变, 进而影响苂光发射. 二者在荧光传感器材 料中有潜在应用。

\section{参考文献}

1 Abraham F, Arab-Chapelet B, Rivenet M, Tamain C, Grandjean S. Coordin Chem Rev, 2014, 266-267: 28-68 
2 Kuhn HJ, Braslavsky SE, Schmidt R. Pure Appl Chem, 2004, 76: 2105-2146

3 (a) Andrews MB, Cahill CL. Chem Rev, 2013, 113: 1121-1136; (b) Cui Y, Yue Y, Qian G, Chen B. Chem Rev, 2012, 112: 1126-1162; (c) Horcajada P, Gref R, Baati T, Allan PK, Maurin G, Couvreur P, Férey G, Morris RE, Serre C. Chem Rev, 2012, 112: 1232-1268; (d) Suh MP, Park HJ, Prasad TK, Lim DW. Chem Rev, 2012, 112: 782-835; (e) Yoon M, Srirambalaji R, Kim K. Chem Rev, 2012, 112: 1196-1231

4 Serezhkina LB, Peresypkina EV, Neklyudova NA, Virovets AV, Serezhkin VN. Crystallogr Rep, 2013, 58: 275-279

5 Ling J, Qiu J, Burns PC. Inorg Chem, 2012, 51: 2403-2408

6 Medrish IV, Virovets AV, Peresypkina EV, Serezhkina LB. Russ J Inorg Chem, 2008, 53: 1034-1039

7 Thuéry P, Rivière E. Dalton Trans, 2013, 42: 10551-10558

8 Thuéry P. CrystEngComm, 2008, 10: 1126-1128

9 Serezhkina LB, Peresypkina EV, Medvedkov YA, Virovets AV, Serezhkin VN. Russ J Inorg Chem, 2013, 58: 1465-1469

10 Serezhkina LB, Marukhnov AV, Peresypkina EV, Virovets AV, Medrish IV, Pushkin DV. Russ J Inorg Chem, 2008, 53: 837-841

11 (a) Serezhkina LB, Peresypkina EV, Virovets AV, Medvedkov YA, Serezhkin VN. Crystallogr Rep, 2014, 59: 48-52; (b) Serezhkina LB, Peresypkina EV, Virovets AV, Neklyudova NA, Pushkin DV. Crystallogr Rep, 2008, 53: 651-654

12 (a) Mihalcea I, Henry N, Loiseau T. Eur J Inorg Chem, 2014, 2014: 1322-1332; (b) Shu YB, Xu C, Liu WS. Eur J Inorg Chem, 2013, 2013: 3592-3595; (c) Wu HY, Ma YQ, Zhang X, Zhang H, Yang XY, Li YH, Wang H, Yao S, Yang W. Inorg Chem Commun, 2013, 34: 55-57; (d) Jouffret LJ, Wylie EM, Burns PC. J Solid State Chem, 2013, 197: 160-165; (e) Nelson AGD, Alekseev EV, Albrecht-Schmitt TE, Ewing RC. J Solid State Chem, 2013, 198: 270-278

13 Wang LH, Shang R, Zheng Z, Liu CL, Wang ZM. Cryst Growth Des, 2013, 13: 2597-2606

14 Rabinowitch E, Bedford RL. Spectroscopy and Photochemistry of Uranyl Compounds. Oxford: Pergamon Press, 1964

15 Dolomanov OV, Bourhis LJ, Gildea RJ, Howard JAK, Puschmann H. J Appl Crystlogr, 2009, 42: 339-341

16 Sheldrick GM. Acta Crystlogr C Struct Chem, 2015, 71: 3-8

17 Bernstein J, Davis RE, Shimoni L, Chang NL. Angew Chem Int Ed, 1995, 34: 1555-1573

18 (a) Buttress GD, Hughes MA. J Chem Soc A, 1968, 1985; (b) Dollimore D, Jones LF, Nicklin T, Spooner P. J Chem Soc Faraday Trans 1, 1973, 69: 1827-1833; (c) Cetişli H, Çılgı GK, Donat R. J Therm Anal Calorim, 2012, 108: 1213-1222

19 (a) Sutton J. Nature, 1952, 169: 235-236; (b) McGlynn SP, Smith JK, Neely WC. J Chem Phys, 1961, 35: 105-116

20 Allen PG, Bucher JJ, Clark DL, Edelstein NM, Ekberg SA, Gohdes JW, Hudson EA, Kaltsoyannis N, Lukens WW, Neu MP, Palmer PD, Reich T, Shuh DK, Tait CD, Zwick BD. Inorg Chem, 1995, 34: 4797-4807

21 (a) Burrows HD, Kemp TJ. Chem Soc Rev, 1974, 3: 139-165; Liu GK, Beitz JV. The Chemistry of the Actinide and Transactinide Elements. 2006. 2013-21 11; (b) Denning RG. J Phys Chem A, 2007, 111: 4125-4143

22 (a) Garg CL, Narasimham KV. Spectrochim Acta Part A-Mol Sp, 1970, 26: 627-635; Burkov VI, Kizel VA, Krasilov YI. Opt Spectrosc, 1976, 40: 469-472; (b) Brittain HG, Perry DL. J Phys Chem, 1981, 85: 3073-3078; (c) Krasovskii AN, Boikov VN, Umreiko DS. J Appl Spectr, 1984, 40: 321-324; (d) Lermontov AS, Lermontova EK, Wang YY. Inorg Chim Acta, 2009, 362: 3751-3755

23 (a) Bukalov SS, Vdovenko VM, Ladygin IN, Suglobov DN. J Appl Spectrosc, 1970, 12: 263-265; (b) Morss LR, Edelstein NM, Fuger J. The Chemistry of the Actinide and Transactinide Elements. Heidelberg: Springer, 2006

24 Liu Y, Chu T, Wang X. Inorg Chem, 2013, 52: 848-854 


\title{
Synthesis, structure and optical properties of two uranyl oxalate complexes templated by protonated amines
}

\author{
Xueling Qiao ${ }^{1}$, Yaxing Wang ${ }^{2}$, Jiangang $\mathrm{He}^{1}$, Wenqi Wei ${ }^{1}$, Rui Ge ${ }^{1}$, Chunli $\mathrm{Liu}^{1 *}$, Shuao Wang ${ }^{2 *}$ \\ ${ }^{1}$ Beijing National Laboratory for Molecular Sciences, Fundamental Science Laboratory on Radiochemistry \& Radiation Chemistry, College of \\ Chemistry and Molecular Engineering, Peking University, Beijing 100871, China \\ ${ }^{2}$ School for Radiological and Interdisciplinary Sciences (RAD-X), Soochow University, Suzhou 215123, China \\ *Corresponding authors (email: liucl@pku.edu.cn; shuaowang@suda.edu.cn)
}

\begin{abstract}
C}_{6} \mathrm{H}_{14} \mathrm{~N}\right]_{4}\left[\mathrm{UO}_{2}\left(\mathrm{C}_{2} \mathrm{O}_{4}\right)_{2}\left(\mathrm{HC}_{2} \mathrm{O}_{4}\right)\right] \mathrm{NO}_{3} \cdot 2 \mathrm{H}_{2} \mathrm{O}(\mathbf{C H A})$ and $\left[\mathrm{C}_{8} \mathrm{H}_{18} \mathrm{~N}\right]\left[\mathrm{UO}_{2}\left(\mathrm{C}_{2} \mathrm{O}_{4}\right)\left(\mathrm{NO}_{3}\right)\right]$ (NNDMCHA) were synthesized by volatile method at room temperature, using $\left[\mathrm{C}_{6} \mathrm{H}_{14} \mathrm{~N}\right]^{+}$and $\left[\mathrm{C}_{8} \mathrm{H}_{18} \mathrm{~N}\right]^{+}$as templates, respectively. The structures and optical properties were characterized by combination of single-crystal X-ray diffraction, PXRD, TG-DSC, IR, Raman, UV-Vis and fluorescence spectra. CHA and NNDMCHA crystallized in monoclinic system, and space group $P 2_{1} / \mathrm{c}$. The equatorial coordination number of CHA and NNDMCHA are 5 and 6 , respectively, forming pentagonal bipyramid and hexagonal pyramid. The lattice water in CHA formed $\mathrm{O}-\mathrm{H} \cdots \mathrm{O}$ hydrogen bonds with the oxygen atoms of the mononuclear anions, resulting in a s-shaped anion chain which extended along the $c$ direction. The protonated amines were linked to the anion chains by $\mathrm{N}-\mathrm{H} \cdots \mathrm{O}$ and $\mathrm{C}-\mathrm{H} \cdots \mathrm{O}$ hydrogen bonds, leading to form the larger hexagon and the smaller parallelogram pores, respectively. The photoflurescence of two complexes significantly decreased when exposure under UV light, showing potential application for fluorescent sensor.
\end{abstract}

Keywords: protonated amine, template, uranyl oxalate complexes, optical property

doi: $10.1360 / \mathrm{N} 032017-00040$ 